\title{
NERVE FIBRE DEGENERATION IN THE BRAIN IN AMYOTROPHIC LATERAL SCLEROSIS
}

\author{
BY \\ MARION C. SMITH \\ From the Neurological Unit of the Medical Research Council, National Hospital for Diseases of the \\ Nervous System, Queen Square, London
}

The purpose of this paper is to report briefly some findings concerning the distribution of degenerating myelinated nerve fibres in the brains of patients who had amyotrophic lateral sclerosis. It is not intended to report in detail the distribution of degenerating fibres throughout the central nervous system in this condition, nor to review the lengthy literature concerning it. In order to establish that the patients did in fact suffer from amyotrophic lateral sclerosis a few sections from the spinal cord and brain-stem showing the classical picture of the condition are included. Further, these sections are used to validate the staining methods used in the brain sections.

The customary concept of amyotrophic lateral sclerosis is of a degenerative process affecting mainly the motor neurones of the central nervous system. The lower motor neurones in the spinal cord are usually affected and, to a variable extent, those of the cranial nerves. The upper motor neurones in the hemispheres may also be affected. In the spinal cord the typical fibre picture is of degeneration of cortico-spinal fibres; there is sometimes also degeneration of spino-cerebellar and other anterior and lateral column fibres.

Cases have been described in which degeneration was found to extend throughout the entire corticospinal system, from the motor cortex through the cerebral peduncle, pons, medulla, and cord. But it is more frequently stated that the degeneration in the cortico-spinal tract is, in most cases, limited to the distal part of the fibres, and that it often cannot be traced further rostrally than the medulla or pons. This was the experience of Davison, who reported in 1941 ( $a$ and b) that in the 37 cases which he had studied he could not find any degeneration above the medulla in 16 cases, nor above the peduncle in 25 cases. On the other hand, Holmes had found degeneration throughout the cortico-spinal system, from cortex to cord inclusively, in all 10 of the 10 cases he reported in 1909. Later workers have tended to neglect both his work and the methods he used.

Apart from the degeneration in the cortex, a little degeneration has been occasionally noted in other parts of the brain. Holmes found a few fibres passing between the main mass of degenerating cortico-spinal fibres in the internal capsule and the lateral nucleus of the thalamus. These degenerating fibres were observed in three of the 10 cases he studied. Other workers do not seem to have confirmed this finding. A very few degenerating fibres have also been occasionally seen in the brain-stem. These observations have never been adequately illustrated nor confirmed; nor have they been generally accepted.

\section{Material and Methods}

The material consists of the central nervous system from seven patients who were diagnosed as suffering from amyotrophic lateral sclerosis.

In the first three cases representative blocks from different regions of the brain and cord were examined. In the last four cases a large series of blocks was taken from the hemispheres. As the first three cases had revealed a wide distribution of degenerating fibres in the cortex it was planned to examine the gyri in subsequent cases with particular reference to their relationship to the motor cortex. After fixation of the brain the pia arachnoid membrane was removed, the precentral gyrus and paracentral lobule were identified, and the surface of these gyri painted with Indian ink. The hemispheres were then sliced and photographed. These photographs with the black outline of the so-called motor cortex have greatly helped in precisely localizing the degeneration in the stained sections.

Many of the blocks were prepared by the Marchi method. Celloidin sections were also prepared and stained by thionin, by haematoxylin and van Gieson's method, by Loyez' method and by the Weigert-Pal method. Frozen sections were stained by the Sharlach $\mathbf{R}$ method. A few sections were also stained by other methods as required. 
General Pattern of Degeneration in Cord and Lower Brain-stem.-The seven cases all came to necropsy diagnosed as amyotrophic lateral sclerosis. In all of them, the distribution of degeneration in the spinal cord was that which enables the pathologist to confirm the clinical diagnosis.

Fig. 1 comprises a series of transverse sections, stained by the Marchi method, taken through the spinal cord and brain-stem of Case 7. There is vivid black staining of the cortico-spinal tracts at all levels. Figs. 2A and B are sections from the same case, stained by the Marchi and Weigert-Pal methods, respectively. In the Marchi preparation many of the myelinated fibres are in a state of active degeneration, as shown by the black staining. In the Weigert-Pal preparation there is pallor of the cortico-spinal tract indicating actual loss of myelinated fibres. The distribution of the region of degeneration is the same in the two sections.

Figs. 3A and B are sections from Case 7, stained by the Marchi and Loyez' methods, respectively. In this case the Marchi section is much more informative than the Loyez. In the Loyez section there is generalized pallor in the lateral and anterior columns, rather more marked in the anterior corticospinal tract on one side. In the Marchi section there is intense degeneration in the lateral cortico-spinal tracts and also in the anterior tract. There is also definite degeneration in the rest of the lateral and anterior columns, in the anterior horns and anterior commissure.

In six of the seven cases there is clear evidence of active degeneration of nerve fibres in the spinal cord. In the seventh case the length of history was exceptionally long (12 years) and the distal part of the cortico-spinal tracts is almost completely degenerated; there is little active degeneration to be seen in the cord or lower brain-stem, although there is in the cerebral hemispheres.

The next few sections illustrate the value of the Marchi method in demonstrating the presence of degenerating fibres, not only in the cortico-spinal tracts, but in regions where the degeneration is less intense.

In the section shown in Fig. 4 (Case 1) there is marked degeneration in the pyramid; there is also degeneration in the hypoglossal nerve, the medial longitudinal fasciculus, and the predorsal fasciculus. There is scattered degeneration in the reticular formation, which is not seen clearly at this magnification.

In the section shown in Fig. 5 (Case 7) which is the nucleus of the seventh cranial nerve, fibres and nerve cells are both seen to be degenerating.

The section in Fig. 6 (Case 7) shows degenerating fibres in the superior colliculus-in the stratum album profundum, and in the tegmentum.

The sections shown so far demonstrate the typical picture of fibre degeneration in amyotrophic lateral sclerosis. Also, they demonstrate the value of Marchi preparations in this kind of material, for it is clearly very much easier to recognize degeneration when the products of degeneration are stained rather than to recognize degeneration by the absence of fibres, as in Weigert-Pal or Loyez preparations. Sections in which degeneration of fibres is shown by lack of staining are adequate to demonstrate marked loss of fibres in a well-defined tract, but are unsatisfactory to demonstrate a less extensive loss of fibres in a tract, especially when the tract is intermingled with fibres of other tracts. It has frequently been noted that it is less easy to identify degeneration in the cortico-spinal fibres in the brain-stem than in the cord in normal myelin preparations. This may be partly due to the frequent, but not invariable, finding that most of the fibres in the caudal part of the internal capsule and further distally are at a more advanced stage of degeneration than those seen at more rostral levels. This demonstrated in Fig. 7. In the caudal part of the capsule (Fig. 7A) the degeneration material is almost all in compound granular cor puscles, in the rostral part (Fig. 7B) it is partly i corpuscles and partly in degenerating nerve fibres $\frac{\Omega}{Z}$ and in the corona radiata (Fig. 7C) it is almost all at the stage of degenerating fibres. When fibres are at an early stage of degeneration this is not revealeg by Weigert-Pal or comparable stains.

Degeneration in Cerebral Hemispheres.-Figs. 9-16 are taken from a coronal series (Case 5) passing from a plane anterior to the precentral gyrus to a plane posterior to it. The precentral gyrus and paracentral lobule are indicated by heavy outlining.

A large number of degenerating fibres can be seen to pass to or from the precentral gyrus and paracentral lobule (Figs. 9 and 12). But the degenerating fibres are not confined to these "motor" gyri. Many degenerating fibres pass into the post-central gyrus (Figs. 10A-C). The fibres are particularly numerous in the more anterior part of the gyrus. A smaller proportion go to the posterior part of the postcentral gyrus and the adjacent parietal gyri (Figs. 10 and 15). In more anterior sections, degenerating fibres can be seen to pass into the frontal gyri (Figs. 13 and 14), lying adjacent to the pre-central gyrus. In all the affected gyri the fibres are most abundant in the parts nearest to the superior surface of the hemisphere. A few degenerating fibres are also present in the upper part of the cingulate gyrus and a very few in the temporal gyri. Most of the degenerating fibres can be seen to run between the 

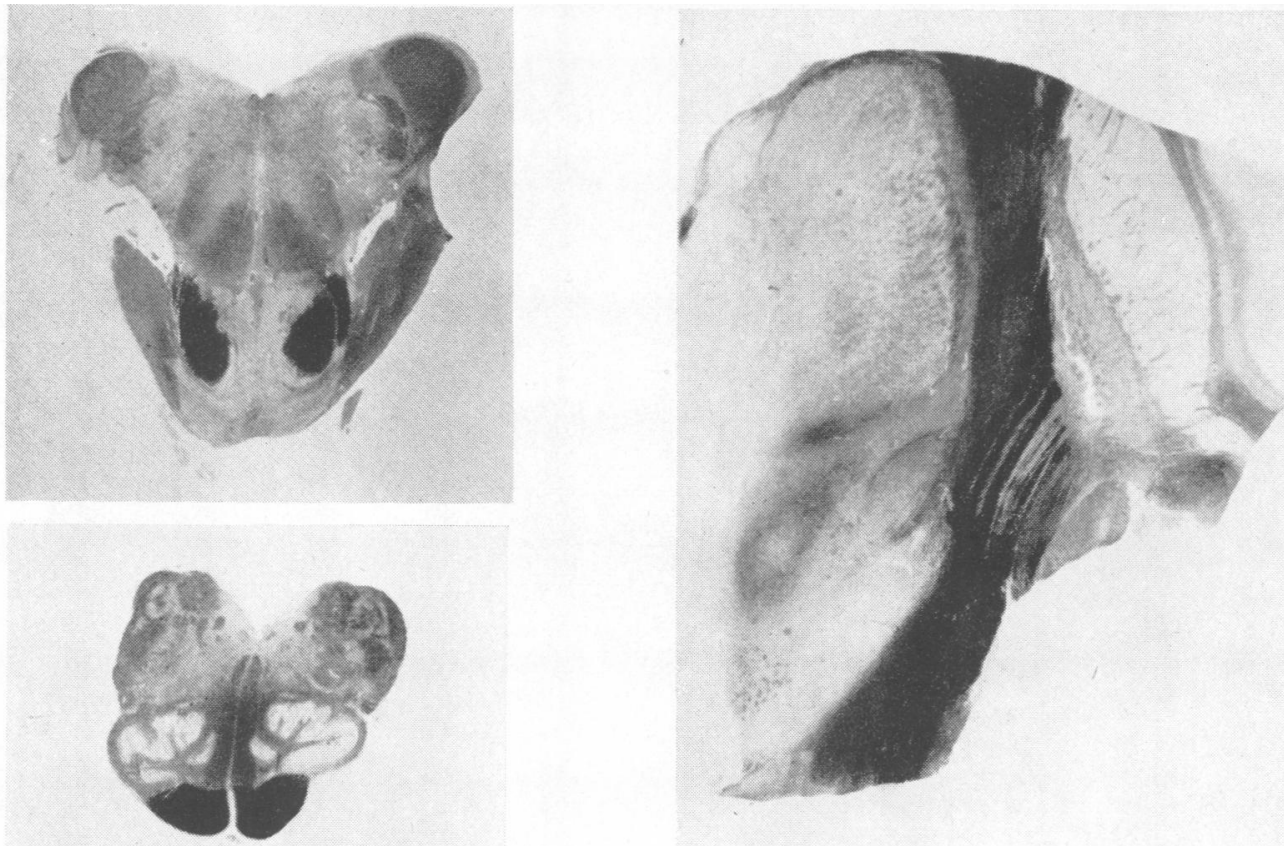

E
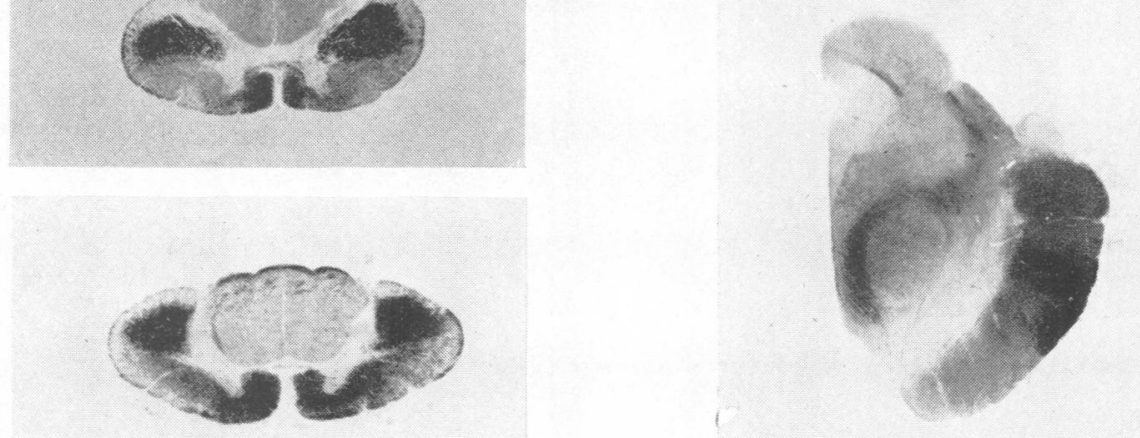

C
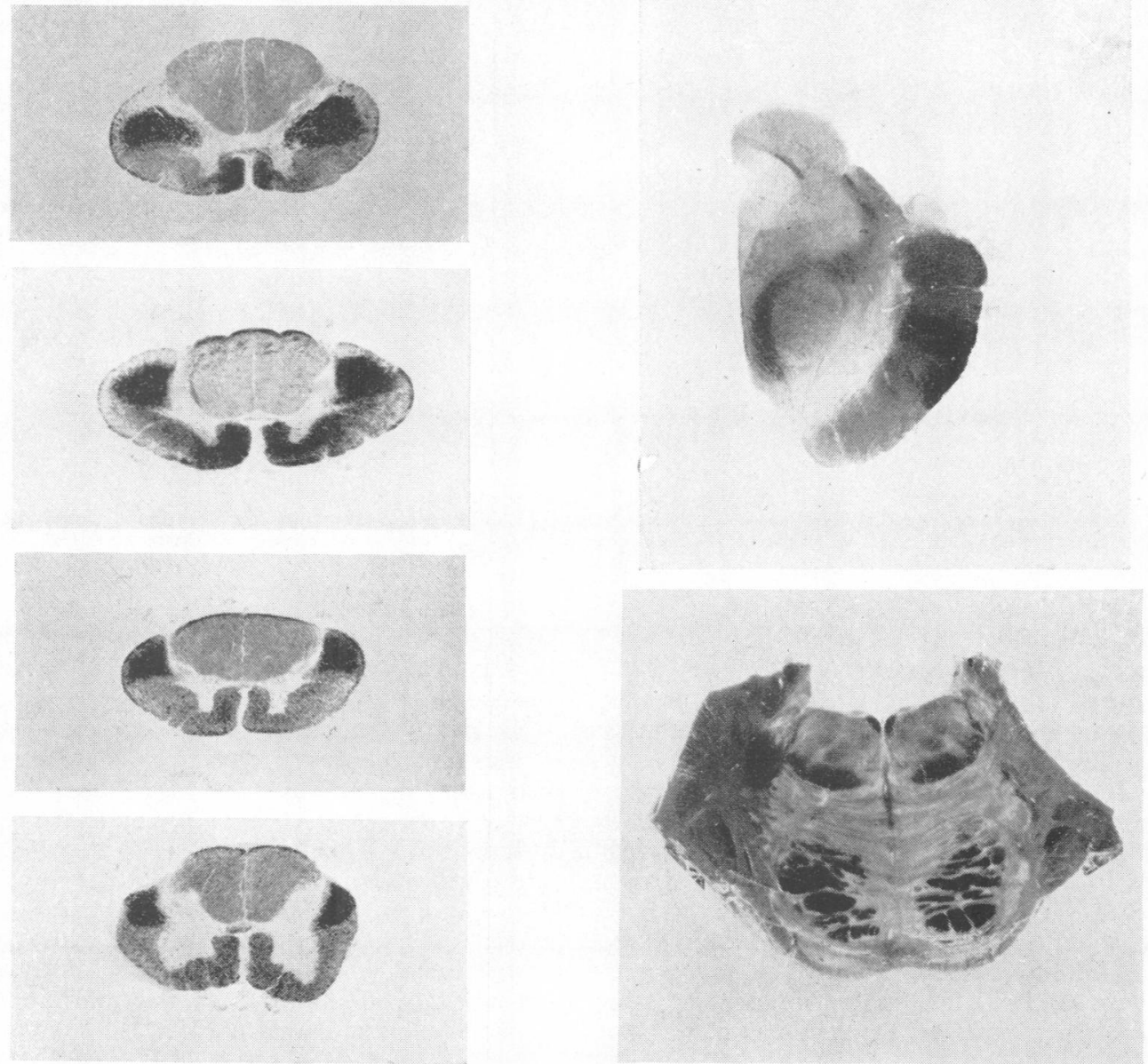

H

A

FIG. 1 (Case 7).-Series of Marchi preparations showing marked degeneration (black staining) of cortico-spinal tracts throughout the cord, medulla, pons, mid-brain, and internal capsule. A-E $\times 3 ;$ F-I $\times 2$. 


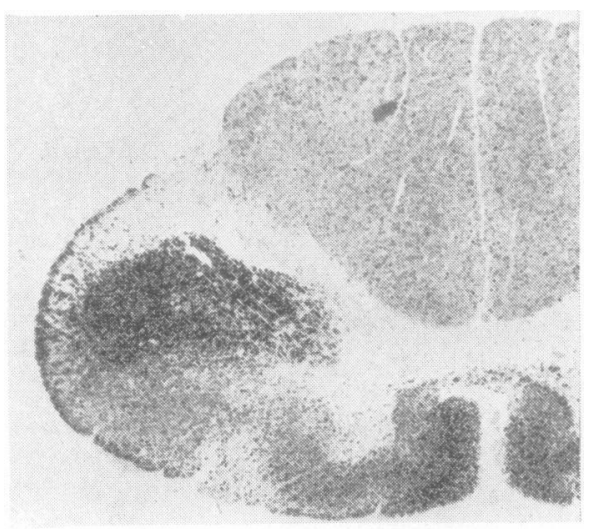

Fig. 2A

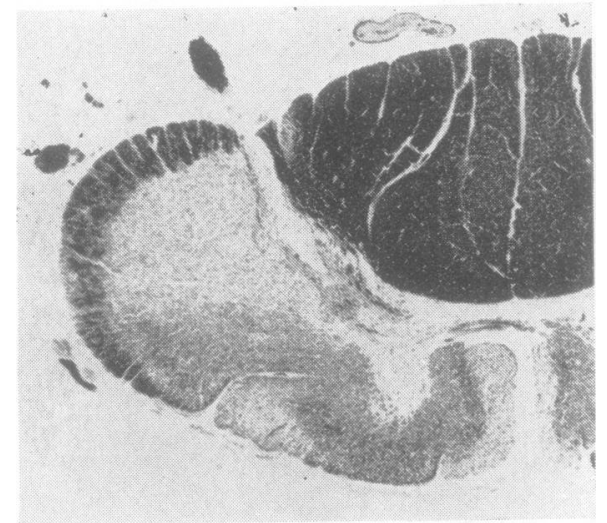

Fig. 2B

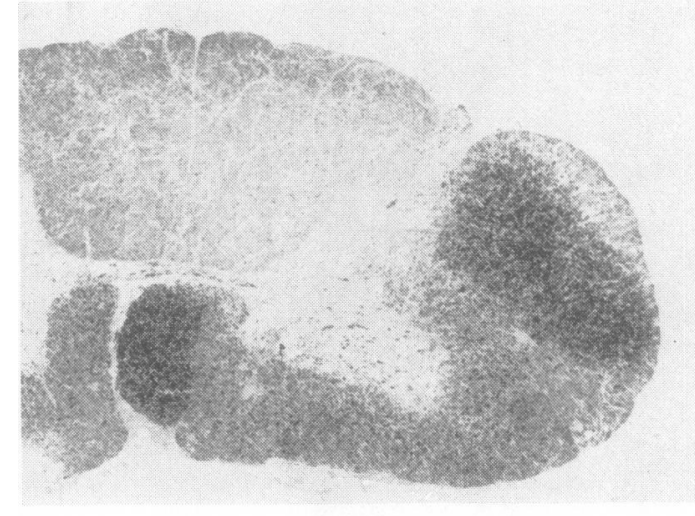

Fig. 3A

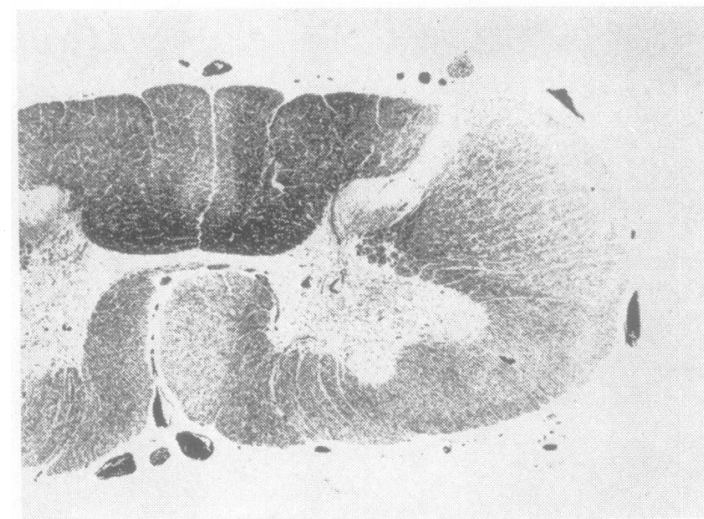

Fig. 3B

Fig. 2 (Case 7).-Cervical cord. A. Marchi preparation $\times 8$. B. Weigert-Pal preparation $\times 8$.

Distribution of degenerating fibres is similar in the two sections, being most marked in the corticospinal tracts. It is shown by black staining in $\mathbf{A}$, and by pallor in $\mathbf{B}$.

Fig. 3 (Case 7).-Cervical cord. A. Marchi preparation $\times 8$. B. Loyez preparation $\times 8$.

Distribution of degenerating fibres is more easily determined in $\mathbf{A}$, in which they are stained black, than in B, where pallor indicates loss of fibres. In A, there is degeneration in the corticospinal tracts, in the ? intrinsic fibres of the anterior and lateral columns, in the anterior horns and anterior commissure.

deeper regions of the corona radiata and the cortex of the gyri. A few fibres run round the base of the sulci between neighbouring gyri. These can be seen in Figs. 9 and 14. These may be collaterals of main fibres, or they may be $U$ fibres.

Many degenerating fibres are present in the corpus callosum. These are most abundant in the middle part (Fig. 11), but they occur also in considerable numbers in the posterior part (Fig. 16) and, to a lesser extent, in the anterior part. It has not been possible, so far, to determine what proportion of the degenerating fibres from any one gyrus passes into the corpus callosum, and what proportion passes into the deeper parts of the white matter. The majority of the fibres undoubtedly enter that part of the internal capsule usually considered to contain cortico-spinal fibres.

The main distribution of the degenerating fibres in the cortical gyri in this case is shown diagrammatically in Fig. 17. The distribution is similar in the other six cases, although the number of fibres varies from case to case.

It seems reasonable to conclude that myelinated fibres occupying the spino-cortical region of the internal capsule come from, or go to, at least part of the frontal, precentral, paracentral, post-central, and parietal cortex. Many of the fibres appear to continue into the spinal cord. 


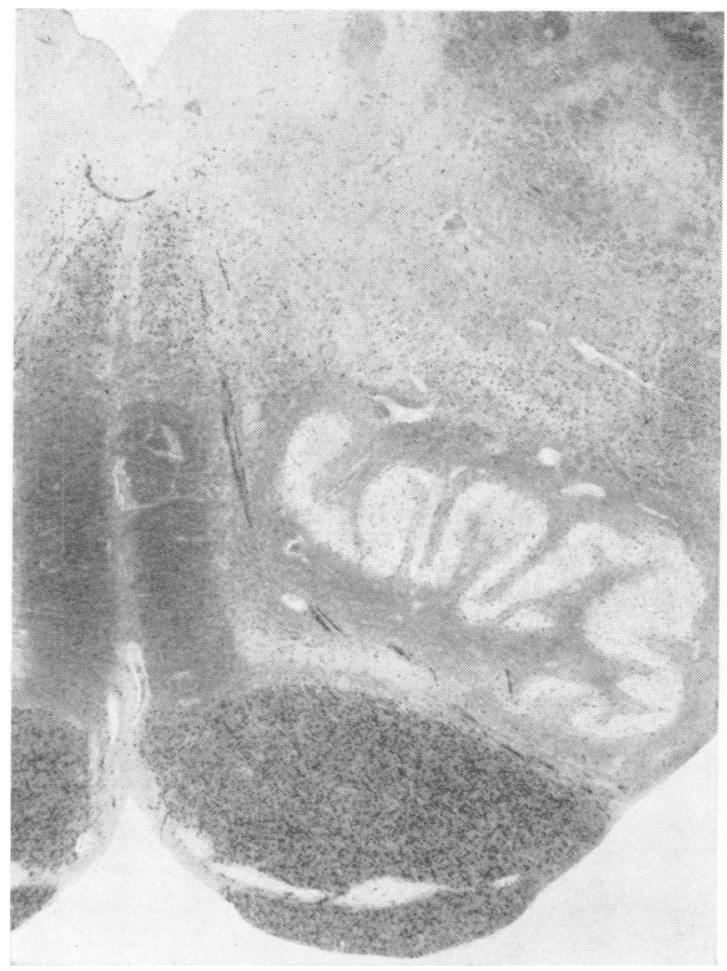

Fig. 4

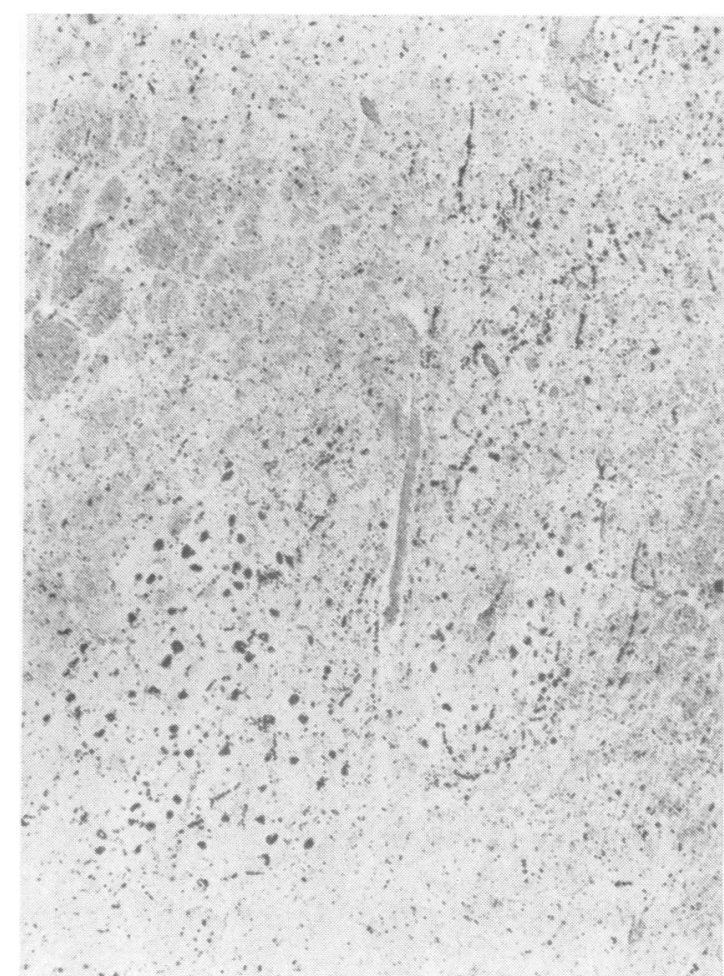

Fig. 5

Fig. 4 (Case 1).-Marchi preparation of medulla, showing degeneration in pyramid, medial longitudinal fasciculus, pre-dorsal fasciculus, and N.XII. There are also degenerating fibres in the reticular formation, not seen clearly at this magnification. $\times 15$.

FIG. 5 (Case 7).-Marchi preparation of pons, showing degeneration in nucleus of N.VII; cells and fibres are both affected. $\times 29$.

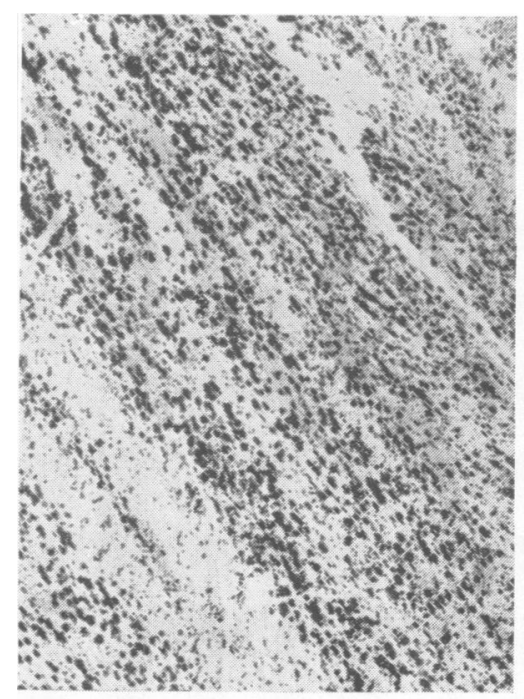

A

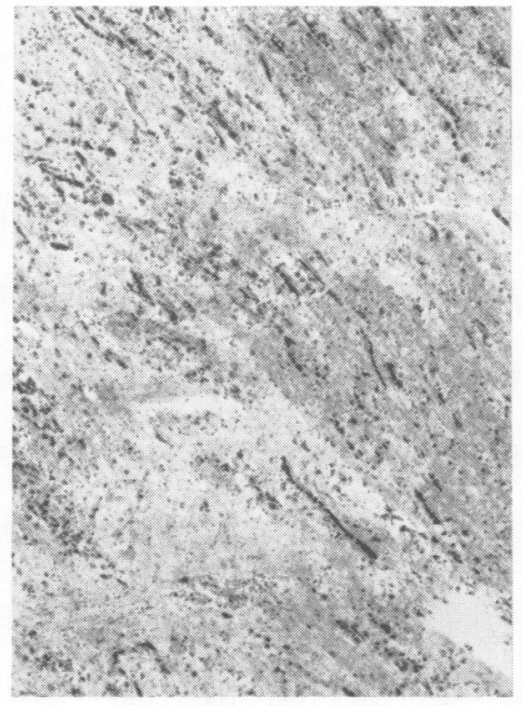

B

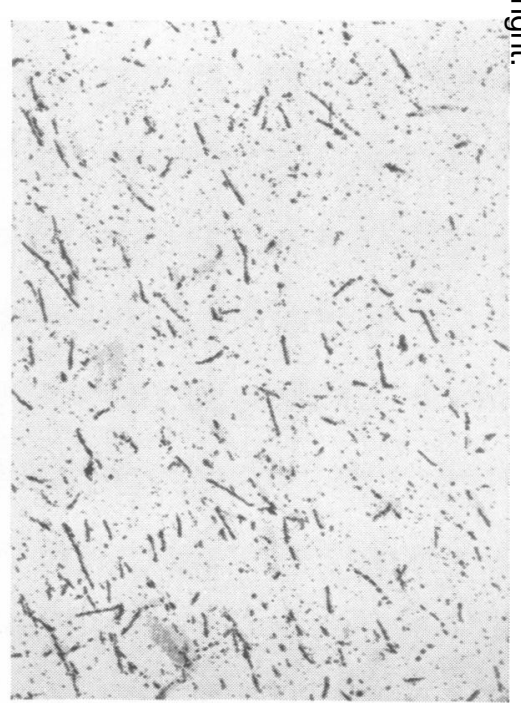

C

Fig. 7

FIG. 7 (Case 2).-Marchi preparation showing different stages of myelin degeneration at three levels of corticospinal tract in the same case. A. Caudal end of internal capsule; degeneration material mainly in compound granular corpuscles. $\times 30$.

B. Rostral end of internal capsule; degeneration material partly in compound granular corpuscles and partly in disrupting myelin sheath stage. $\times 30$.

C. Corona radiata; degeneration material almost entirely in disrupting myelin sheath stage. $\times 30$. 


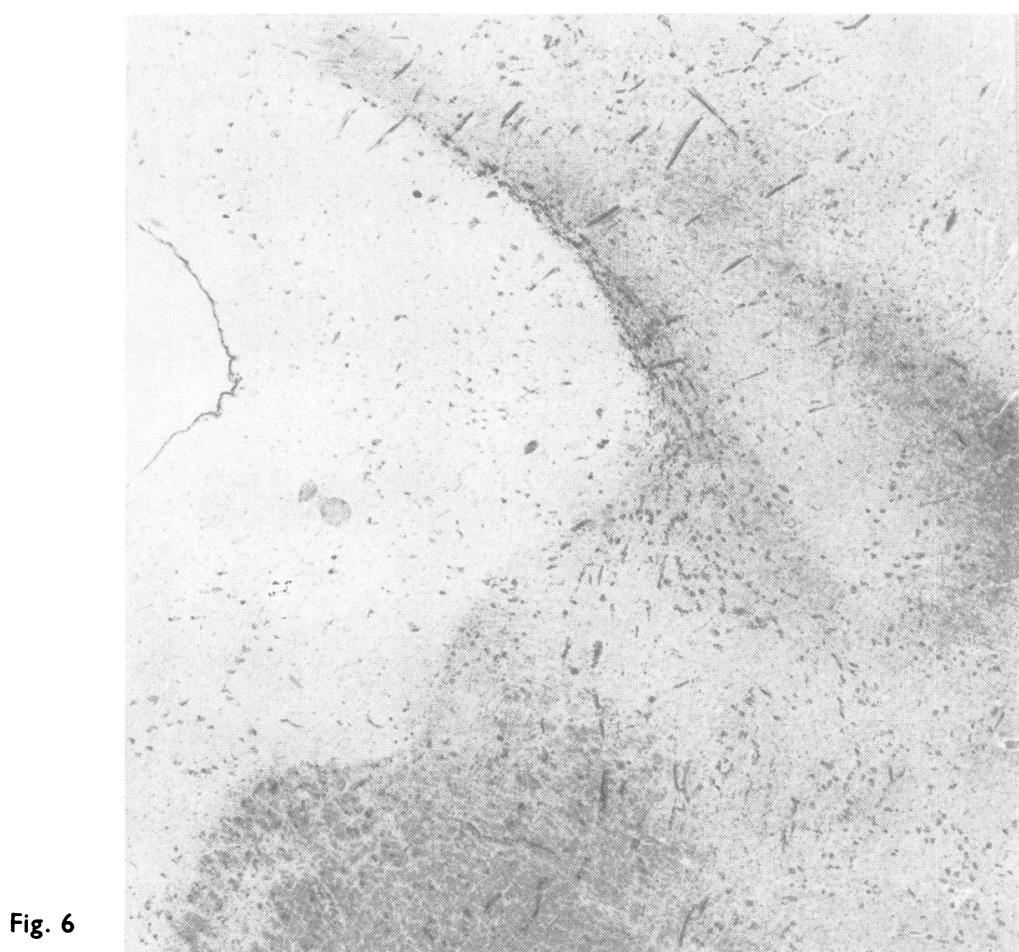

FIG. 6 (Case 7) - Marchi preparation of superior colliculus (Case 7) showing degenerating fibres in the colliculus, in the stratum album profundum, and in fibres in the tegmentum (? efferent collicular fibres). $\times 14$.

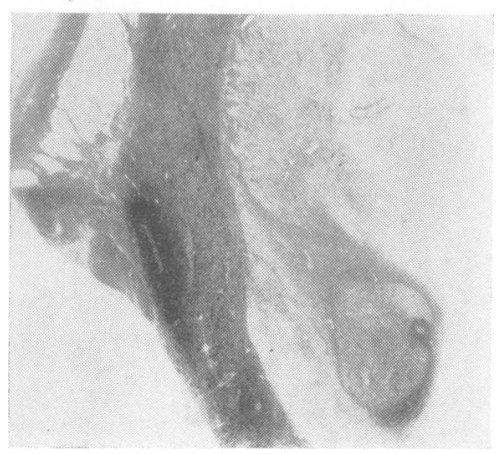

Fig. 8A

Fig. 8 (Case 2).-Marchi preparation of internal capsule and thalamus showing degenerating fibres passing between the capsule and the lateral nucleus of the thalamus. $\times 24$.

Fig. 8B

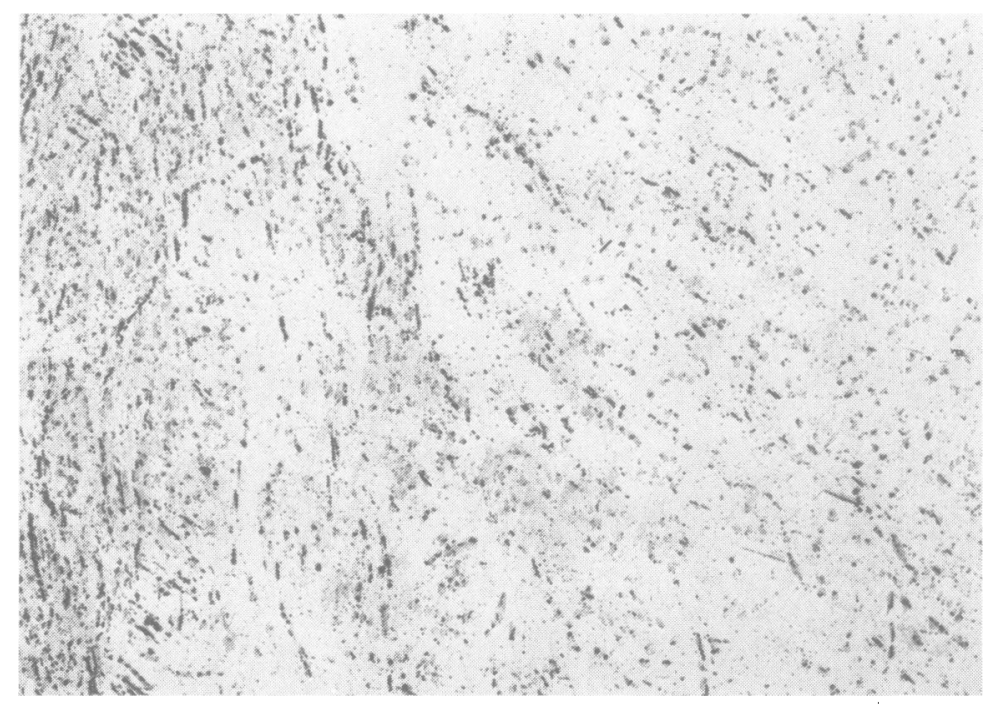




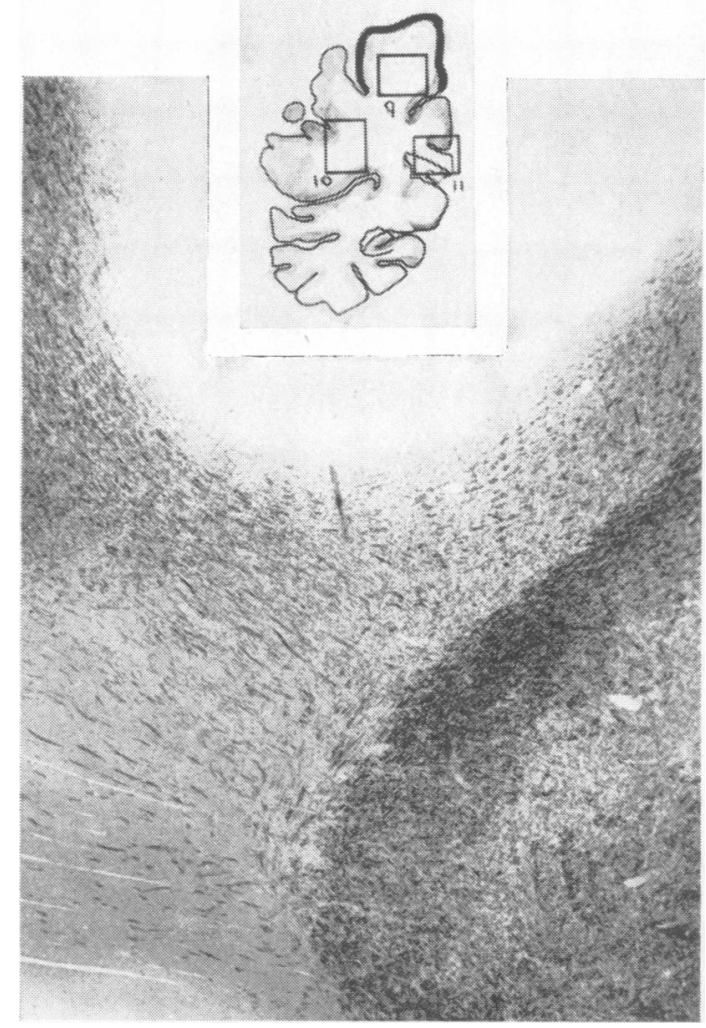

Fig. 10A

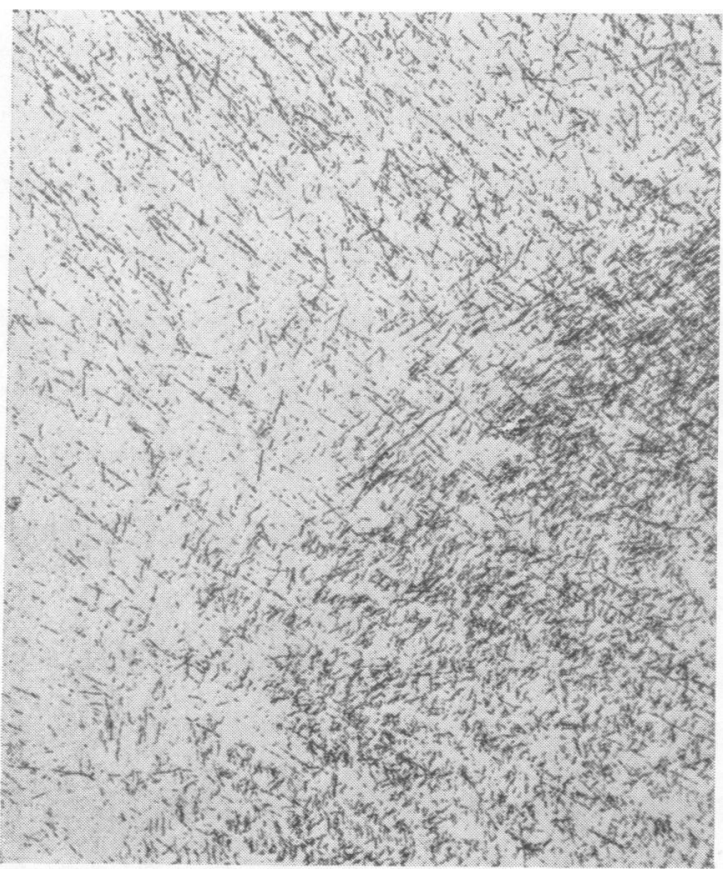

Fig. 10B

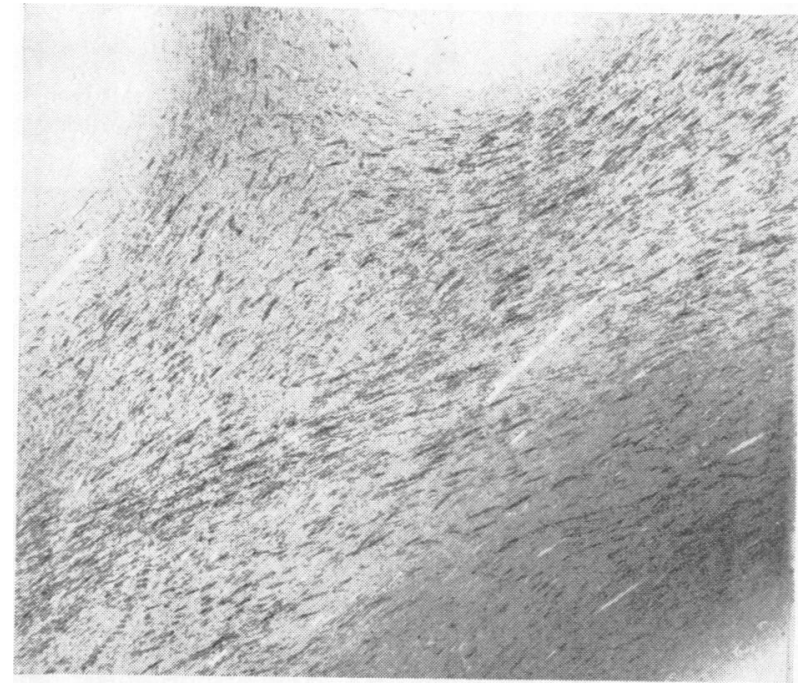

Fig. 9
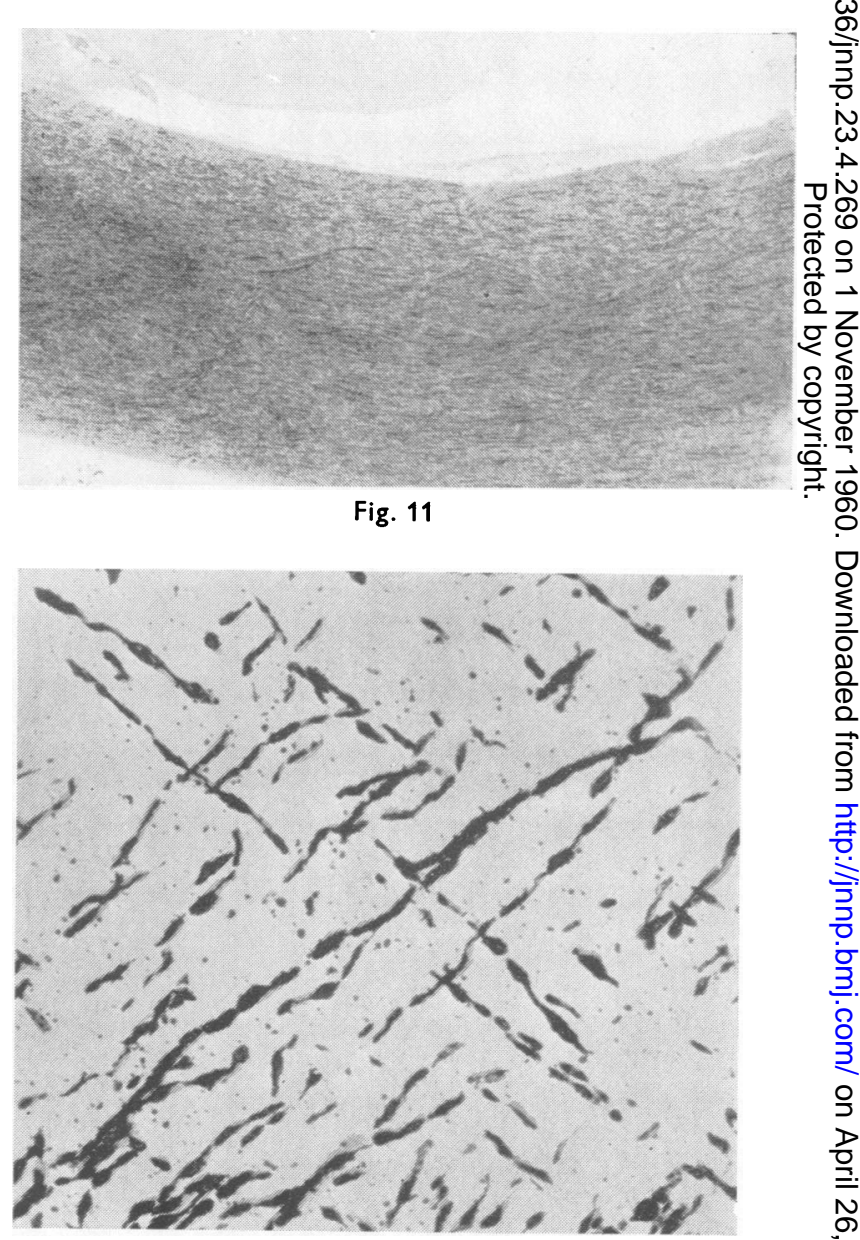

Fig. 10C

Figs. 9-16 (Case 5).-Marchi preparation from the cerebral hemisphere showing distribution of degenerating fibres. The pre-central gyrus and paracentral lobule are heavily outlined in the key diagrams.

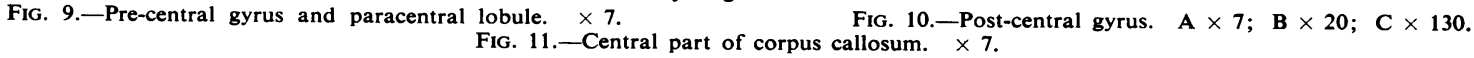




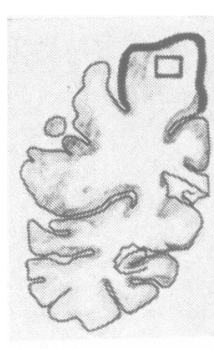

Fig. 12

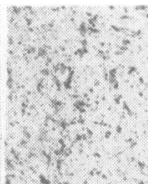

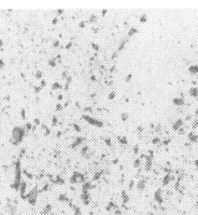

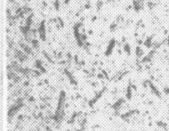

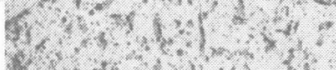

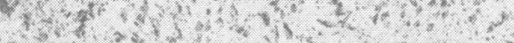

$(1+3)+2 x+2$

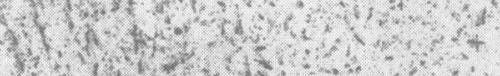

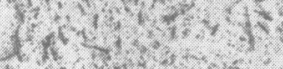

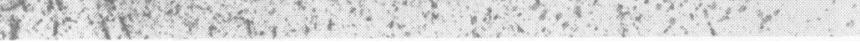

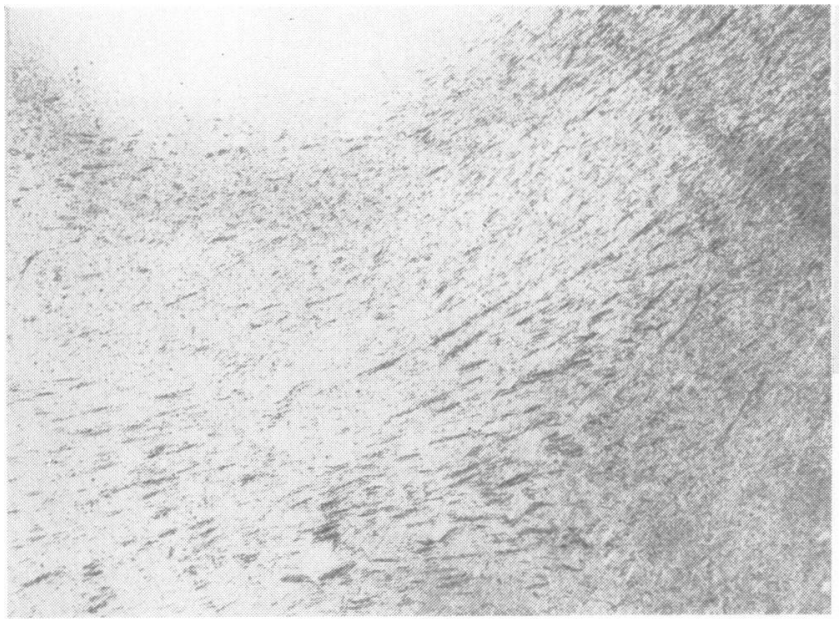

Fig. 13

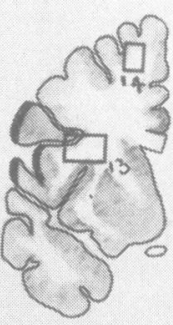

Fig. 14

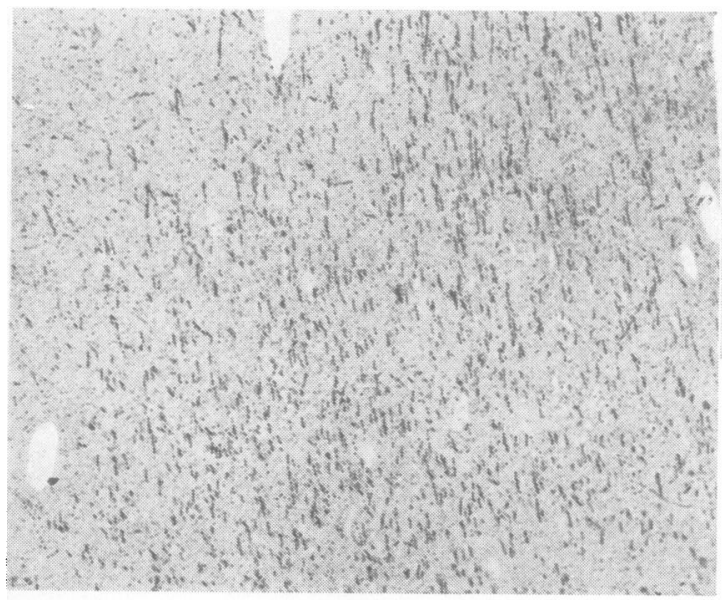

Fig. 15

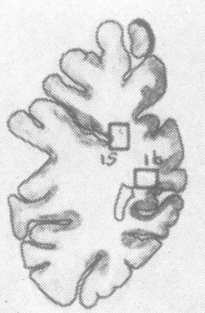

Fig. 15.-Parietal lobe. $\times 20$
Fig. 12.-Cortex of pre-central gyrus. $\times 20$ Fig. 14.-Superior frontal gyrus. $\times 20$.
FIG. 16.-Posterior part of corpus callosum. $\times 20$.

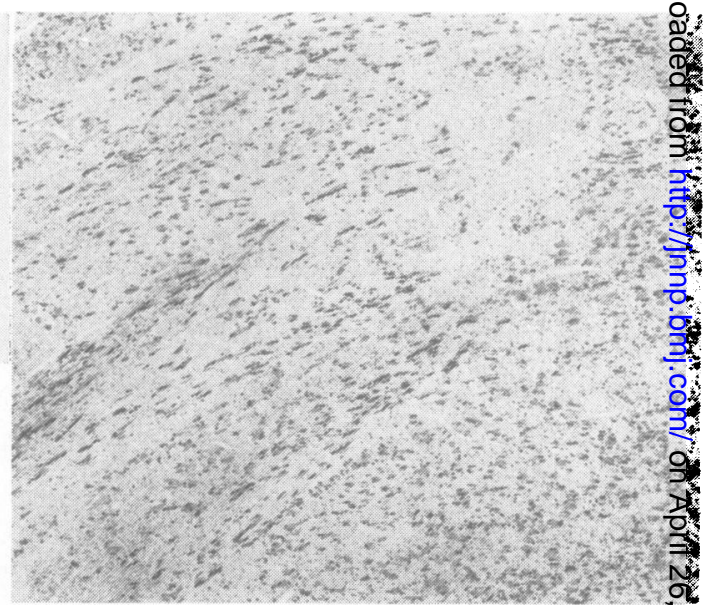

Fig. 16 

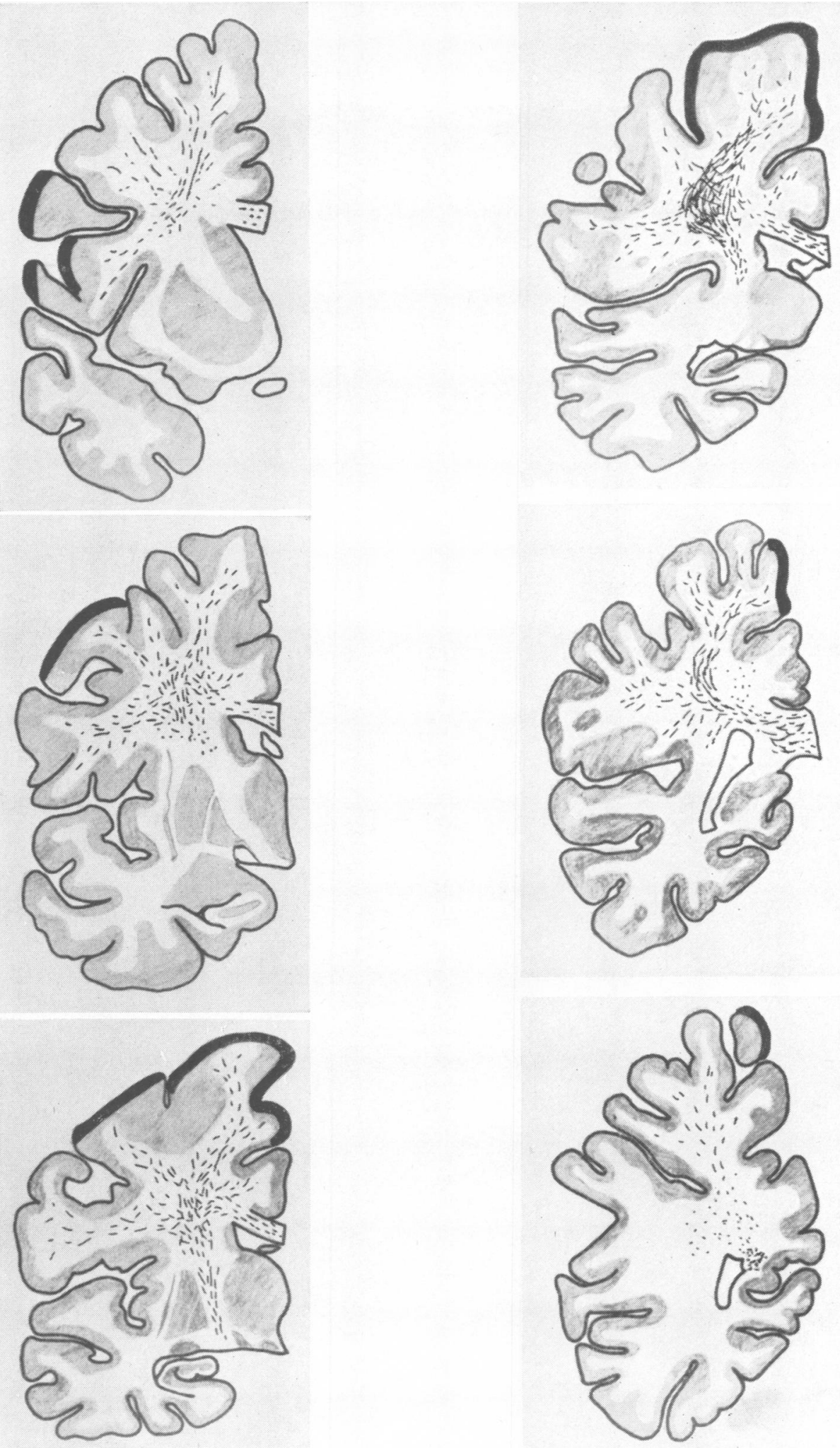

Fig. 17 (Case 5).-Series of diagrams of coronal sections showing distribution of the degenerating fibres. The precentral gyrus and paracentral lobule are heavily outlined. 


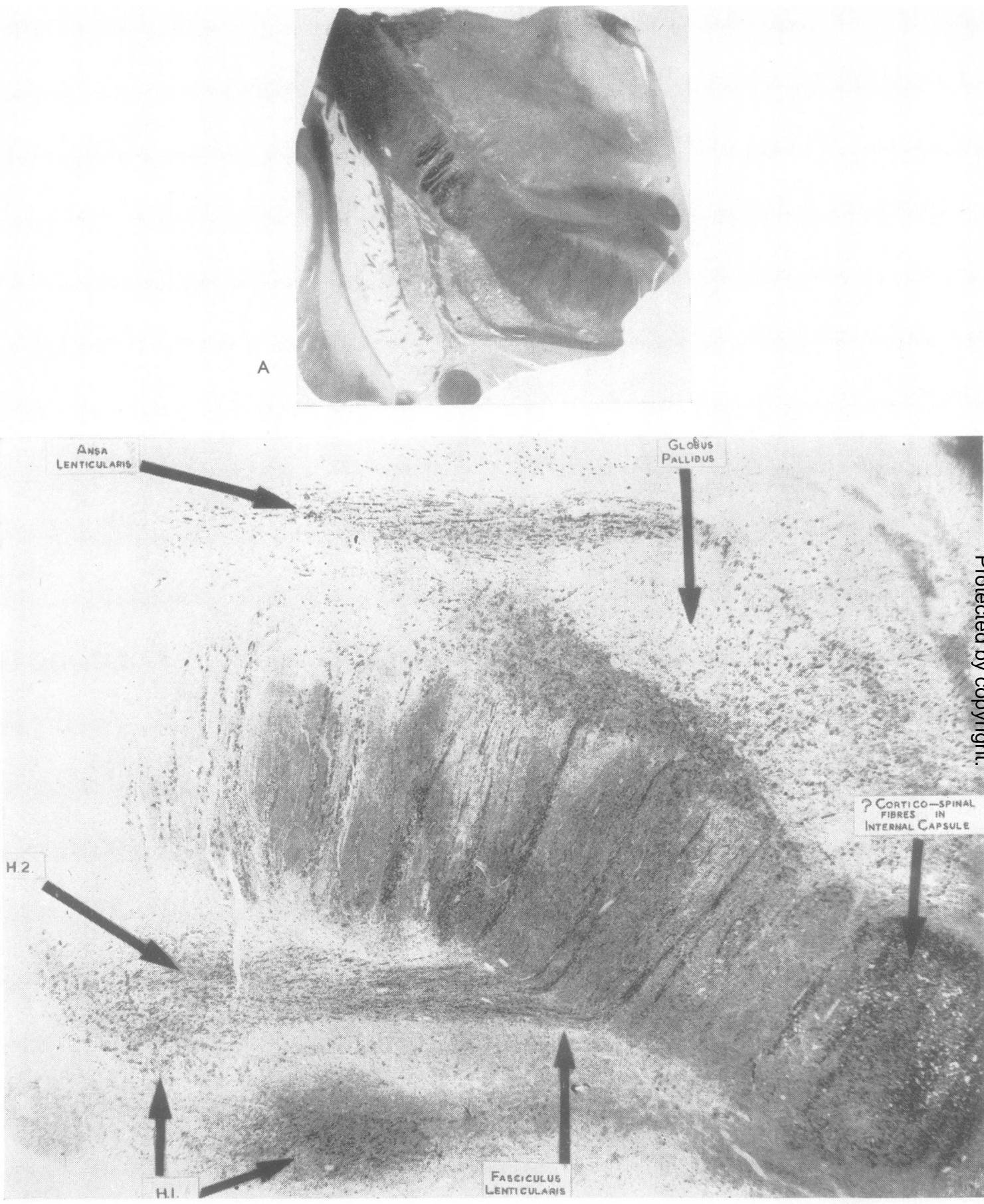

B

FIG. 18 A-D. (Case 3)-Marchi preparation of horizontal section through internal capsule and basal ganglia. There is a concentrated group of ? cortico-spinal degenerating fibres in the capsule. Numerous degenerating fibres are also in the globus pallidus, the ansa lenticularis, the fasciculus lenticularis, and $\mathrm{H}_{2}$. A few fibres, $\mathrm{H}_{1}$, pass from $\mathrm{H}_{2}$ into the thalamus. A $\times 2 ; B \times 7 ; C$ and $D \times 16$. 

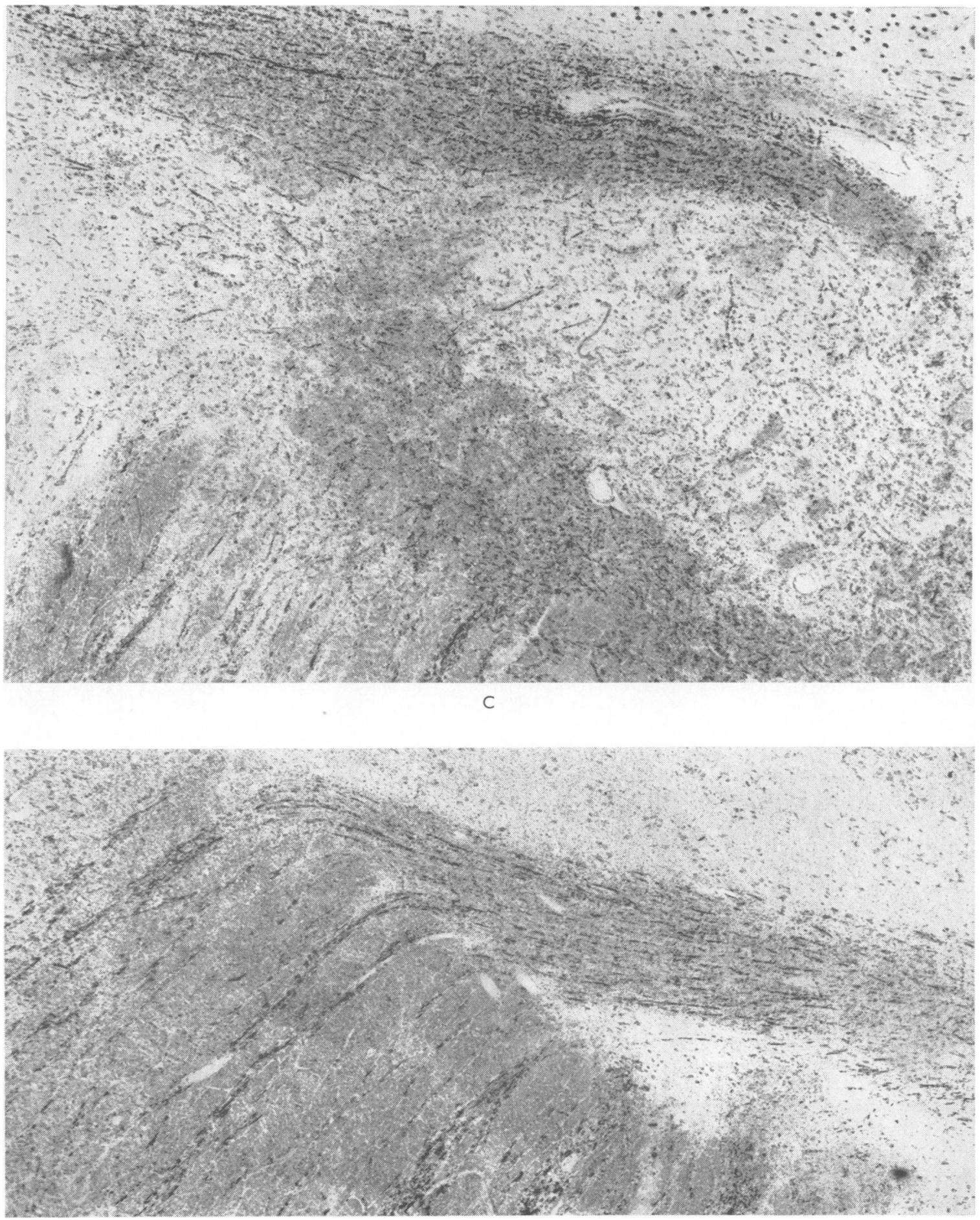
Degeneration in Thalamus, Basal Ganglia, and Peduncular Nuclei.-The next observation to be considered is the presence of degenerating fibres in other parts of the cerebral hemispheres.

In all the cases degenerating fibres run between the main mass of degenerating fibres in the internal capsule and the lateral nucleus of the thalamus (Fig. 8, Case 2).

Degenerating fibres are present in the basal ganglia in four of the cases. In the other three cases this region was not examined until after prolonged fixation, and the absence of degenerating fibres in the Marchi preparations in these cases may be due to this factor only (Smith, 1956).

The degenerating fibres are mainly in the globus pallidus, and in the ansa lenticularis and fasciculus lenticularis. (These terms are used in the same sense as by Papez, 1942.) In horizontal sections through the lentiform nucleus, internal capsule and thalamus, the degenerating fibres can be seen in the bundles which compose the fasciculus lenticularis, passing through the internal capsule, and in the ansa lenticularis, as it passes round the anterior part of the posterior limb of the capsule (Fig. 18, Case 3). The fibres of the two groups can be traced in adjoining sections until they unite forming the bundle $\mathrm{H}_{2}$. The fibres of $\mathrm{H}_{2}$ can be traced towards the pre-rubral field (Forel's field $\mathrm{H}$ ). From $\mathrm{H}_{2}$ a number of fibres, $\mathrm{H}_{1}$, go to the ventrolateral nucleus of the thalamus, and a few fibres go to the hypothalamus. Some of the fibres perforating the internal capsule pass into the capsule of the subthalamic nucleus and a number of degenerating fibres are scattered through this nucleus.

In three of the cases a few degenerating fibres are present in the substantia nigra.

As mentioned earlier, degenerating fibres are scattered about in the reticular substance of the mid-brain, pons, and medulla. In the mid-brain there are, in all cases, degenerating fibres in the group round the aqueduct and in the superior colliculi. It is possible that there is some connexion between some of these degenerating fibres and the groups of degenerating fibres in the basal ganglia. But precisely which fibres are degenerated, and their course and connexions have not been worked out yet.

In all regions in which degenerating fibres have been described in Marchi preparations, Sharlach $\mathbf{R}$ preparations have shown the presence of fat, although in far less abundance than the degeneration products demonstrated by the Marchi method; and in some of the regions there has been distinct gliosis.

\section{Discussion}

A point which seems of interest is that in all the seven cases of amyotrophic lateral sclerosis which were examined there was clear and abundant degeneration in the cortico-spinal tracts at all levels, from cortex throughout the brain-stem and cord. This is in accordance with the findings of Holmes (1909) in all of his 10 cases. But the amount of degeneration was much more abundant in several of the cases here than that described by Holmes, and, as discussed below, it was much more extensive. In looking for degeneration in these brains the need for using stains which demonstrate fibres in a state of active degeneration cannot be stressed too strongly. It is relevant that Swank and Putnam (1943) found, from doing fibre counts, that in the anterior nerve roots of patients with amyotrophic lateral sclerosis, up to $50 \%$ of the larger fibres may be lost before ordinary microscopic methods reveal any defect, and they thought that this is true for cortico-spinal fibres also. Their work shows the difficulty in recognizing the loss of even considerable numbers of fibres from tracts, by means of staining the healthy fibres.

Most workers who studied the distribution of pathological changes in the cerebral cortex in this condition were concerned with changes in cortical neurones. Some workers have found the changes to be more extensive in distribution than the socalled motor cortex. The changes which Davison (1941 a and b) found in the cortex of some of his cases affected not only the motor cortex, but the frontal, pre-motor, and opercular areas also. This was confirmed in these same cases by Friedman and Freedman (1950). Holmes (1909), on the other hand, found the changes to be limited to the precentral gyrus and paracentral lobule, and to the part of the post-central gyrus that forms the posterior wall of the fissure of Rolando; the rest of the cortex was invariably free from degeneration. It is of interest that the area in which Holmes found changes is coextensive with the area which he and May (1909) established as being the site of origin of cortico-spinal fibres.

Some later workers have shown in monkeys that cortico-spinal fibres arise from a considerably more extensive region of the cortex than the classical motor strip, although there is no general agreement about the origin of the fibres. Fibres have been described as arising from that part of the frontal lobe immediately anterior to the pre-central gyrus (Minkowski, 1923, 1924 a and b; Uesugi, 1937), and from the post-central gyrus (Levin and Bradford, 1938) and from the adjacent part of the parietal cortex also (Peele, 1942, 1944). These findings in monkeys give support to the view that the bulk of the degenerating fibres seen in the subcortical white matter, in the present series of cases, belong to the cortico-spinal system. 
The presence of degenerating fibres in the thalamus was noted by Holmes (1909) in three out of his 10 cases. It is present in all seven of this series. It seems likely that degeneration in fibres passing into or out of the thalamus could be demonstrated in most cases of amyotrophic lateral sclerosis if the appropriate stains were used. It is not possible at present to say whether these are corticopetal or corticofugal fibres, or to determine with which region of the cortex they are connected.

In the four cases examined soon after fixation there was definite degeneration in the ansa lenticularis and fasciculus lenticularis. It is possible that degeneration would have been revealed in the other three cases had they been examined under optimal conditions. Degeneration in the basal ganglia has seldom been described in cases definitely shown to be amyotrophic lateral sclerosis, although Bertrand and van Bogaert (1925) did find some pallor in the fibres of the ansa lenticularis in some of their cases.

It was impossible to trace completely the course of the degenerating fibres from, or to, the basal ganglia, the substantia nigra, tegmentum and colliculi, but the course of many of the ansa and fasciculus lenticularis fibres could be followed. If degeneration occurs as frequently in the basal ganglia and peduncular nuclei as would appear from the findings in these cases, then detailed studies made on material from patients with amyotrophic lateral sclerosis may yield valuable information about anatomical relationships in the human brain.

It is not possible in the material examined so far to say precisely in which nuclei the degenerating fibres take origin and terminate. But in view of the distribution of the fibres in the ansa lenticularis, the fasciculus lenticularis, the field of Forel, the thalamus and hypothalamus, it is most probable that many arise in the globus pallidus, for there is good evidence that the efferent pallidal fibres are distributed to these regions (Papez, 1942). If this is indeed so, the involvement of the globus pallidus may contribute to the severity of the muscle paralysis in amyotrophic lateral sclerosis, for Whittier and Mettler (1949) found in monkeys that paresis resulting from damage to the internal capsule or cerebral peduncle was much more severe when combined with destruction of the pallidum or its efferent pathway. In their material, of course, the lower motor neurones were intact, so the effect of a lesion of both internal capsule and pallidum could be more clearly determined than in cases of amyotrophic lateral sclerosis.

Up to 50 years ago it was customary to utilize diseased brains in working out anatomical relationships in man. But several factors have resulted in a decline in this custom. The development of the use of experimental animals, with the possibility of making controlled lesions, at an optimum period before death, has superseded the use of human brains with extensive "natural" lesions. Also, once a histological picture of the disease is established, there is a tendency to examine the tissue only to the extent of confirming the clinical diagnosis and demonstrating the classical picture. It is possible that essential information relevant to the functional anatomy of the human central nervous system may be derived from the detailed study of "routine" neuropathological material, apart from the possibility of making observations contributing to an understanding of the aetiology of the disease.

The present seven cases have been presented as an example of the value of studying such "classical" diseases. This is a preliminary report of degeneration studies which are being made on a variety of conditions, using the staining methods usually applied mainly to experimental material. The observations reported here do not pretend to give a complete picture of the degeneration in amyotrophic lateral sclerosis, but to present some unappreciated aspects of the degeneration in myelinated fibres. There are considerable limitations imposed on drawing deductions from this type of material, in which there is a possible widespread disease process, because we cannot say categorically that the degeneration starts here and goes there, as we can about the degeneration resulting from circumscribed lesions. Nevertheless, it seems that there is evidence that further work utilizing pathological material for anatomical studies may be of definite value.

\section{Summary}

The degeneration of myelinated nerve fibres in the brain in seven cases of amyotrophic lateral sclerosis is described. Degenerating myelinated nerve fibres are present from cortex throughout the brain-stem and cord in every case.

The distribution of the degenerating fibres in the cortex is extensive. These fibres are present not only in the pre-central gyrus and paracentral lobule, but they are also abundant in the postcentral gyrus, and occur in considerable numbers in the adjacent parietal and frontal gyri. A few degenerating fibres are also present in other parts of the cortex.

The majority of these, degenerating fibres appear to pass into the region ascribed to the cortico-spinal system in the brain-stem and to continue into the cord.

Numerous degenerating fibres are present in the corpus callosum. 
Degenerating fibres pass between the main group of degenerating fibres in the internal capsule and the lateral nucleus of the thalamus.

Degenerating fibres are present in the basal ganglia, in particular in the ansa lenticularis and fasciculus lenticularis.

Degenerating fibres are also present in the substantia nigra, the tegmentum and reticular formation of the brain-stem.

The value of the above findings with respect to anatomical studies is discussed.

I am greatly indebted to Professor Blackwood and Dr. Mair for their generosity with the cases reported here and their sustained interest in the problem. I wish to thank also Dr. Carmichael for all the help and facilities he has given me. Miss Ebborn is responsible for most of the histology and Mr. Frampton for the photography. My warmest thanks are due to them.

\section{REFERENCES}

Bertrand, I., and Bogaert, L. van (1925). Rev. neurol., 321, 779. Davison, C. (1941a). Trans. Amer. neurol. Ass., 67, 67.

- (1941b). Arch. Neurol. Psychiat. (Chicago), 46, 1039.

Friedman, A. P., and Freedman, D. (1950). J. nerv. ment. Dis., 111, 1 .

Holmes, G. (1909). Rev. Neurol. Psychiat., 7, 693.

- , and May, W. P. (1909). Proc. roy. Soc. Med., 2, (Neurol. Sect.), 92.

Levin, P. M., and Bradford, F. K. (1938). J. comp. Neurol., 68, 411. Minkowski, M. (1923). Schweiz. Arch. Neurol. Psychiat., 12, 71 and 227 . (1924a). Ibid., 14, 255.

- (1924b). Ibid., 15, 97.

Papez, J. W. (1942).'The Diseases of the Basal Ganglia. A.R.N.M.D., Vol. 21, p. 21

Peele, T. L. (1942). J. comp. Neurol., 77, 693.

(1944). J. Neurophysiol., 7, 269.

Smith, M. C. (1956). J. Neurol. Neurosurg. Psychiat., 19, 67.

Swank, R. L., and Putnam, T. J. (1943). Arch. Neurol. Psychiat. (Chicago), 49, 151

Whittier, J. R., and Mettler, F. A. (1949). J. comp. Neurol., 90, 319.

Uesugi, M. (1937). Anat. Anz., 84, 179. 